\title{
You've Covered: Designing for In-shift Handoffs in Medical Practice
}

\author{
Yunan Chen ${ }^{1,2}$ \\ ${ }^{1}$ Department of Informatics \\ ${ }^{2}$ Institute for Clinical and Translational Science \\ University of California, Irvine \\ Irvine, CA 92697-3440, USA \\ yunanceics.uci.edu
}

\begin{abstract}
Handoffs are moments of critical transition in which clinicians engage to maintain continuous coverage of patient care. This paper reports on an observational study of continuous coverage in an Emergency Department (ED), where three types of handoffs that occur during the same shift were identified: lunch breaks, ad hoc breaks and high workloads. The findings show these "in-shift handoffs" are managed not only through temporal linear coordination, but also through the local coordination among nurses working nearby. In-shift handoffs are crucial to maintaining continuous coverage in hospital settings. However, insufficient understanding of in-shift handoffs in Electronic Medical System (EMR) design may lead to a separation of information and responsibility, and an illusion of communication in patient care. The findings of this study call for attention to in-shift handoffs in future system design and for improving the traditional handoff process through the coordination of local awareness during ED work.
\end{abstract}

Keywords: In-shift Handoffs, Electronic Medical Record (EMR), Emergency Departments, Non-working Moments, Design.

\section{Introduction}

One unique practice that distinguishes hospital work from other domains of health care is the notion of "continuous coverage" [1]. In his work regarding the temporal organization of medical care, Zerubavel writes, "since the hospital's raison d'être is patient care, and since patients require that care regardless of the time of the day, the day of the week, or the time of the year (that is, even at night, on weekends, and on holidays), hospitals must always be open and provide medical and nursing coverage on a continuous basis. [1: P40]." Indeed, hospital service can never stop, yet no employee can work continuously without breaks. Continuous coverage in a hospital is maintained through the rotation of working/non-working times amongst a group of clinicians. In most hospital departments, nurses and doctors rotate their work to provide non-stop service to patients. At the Emergency Department (ED) where this study was carried out, nurses are required to stay at their patients' bedside constantly in order to monitor potentially unstable situations. In other words, continuous 
coverage requires that the end of one shift continue immediately with a new shift, and that an absence of even a few minutes needs to be covered by other nurses.

The notion of continuous coverage raises critical considerations regarding the design of information systems in hospital environments, since the design needs to consider not only the work performed through the system, but also how different nonworking activities are handled. The most studied non-working activity is perhaps the end of shifts handoffs. Handoff is defined as when "two or more workers exchange mission-specific information, responsibility, and authority for an operation [2]." Handoffs bring interruptions into the continuous care process since the "baton" of patient care can easily be dropped during these moments of transition [3][4].

Shift changes are the most common handoffs in hospital work, in particular, those for nursing shifts in inpatient units e.g. [5][6]. Yet, continuous coverage in medical work may involve other kinds of handoffs depending on the urgency of the medical service. As Zerubavel mentions, "having direct responsibility for patients, house staff and nurses never leave their service even for a short time period such as a lunch break without having some 'cover' for them [1: P42]." A lack of consideration for these other types of non-working activities in clinical systems design would inaccurately reflect the work practice being conducted in hospitals.

To understand how continuous coverage is maintained in hospital work, we studied work practices in an Emergency Department (ED). ED patients are often in unstable and life-threatening situations, and even a few minutes of delay in giving medications can be crucial and lead to patient sufferings and care deterioration. Thus, the nonwork activities in an ED are not only limited to shift changes, but also include various breaks that occur during the same shift of work. In this paper, we refer to the handoffs that happen during the same shifts as In-shift Handoffs. We identify three types of in-shift handoffs and discuss how they are coordinated among ED clinicians. These in-shift handoffs, however, have not yet been reported in current HCI/CSCW literatures. The findings of this study provide valuable insights for designing information systems that support work practice in urgent, life-critical, and timeconstraint environments and suggest how information systems can be designed to support the working/non-working moments transitions to better facilitate the practice of continuous coverage in hospital work.

\section{Related Work}

It is commonly believed in HCI field that understanding work practices and user behaviors is key to the design of systems situated in the real working environment. One critical issue in medical work is that it is highly collaborative and often coordinated among multiple clinicians. Hence, researchers in the HCI/CSCW field have long been interested in studying collaboration and coordination in hospital work. Previous studies have suggested that articulating work is essential to successfully ensuring clinical collaboration and coordination [7]. In particular, hospital work is often managed through temporal coordination [8] and spatial coordination [9].

Maintaining continuous coverage is certainly a coordination issues and it is often studied in the context of shift changes handoffs. A handoff process contains the transition of not only information, but also responsibility and authority between two or more people [2]. Handoffs are often considered as "drop points" in continuous patient 
care since the transition of care often occurs within short time periods and under pressure [10]. Medical literatures show that handoffs carry a high risk of communication and information breakdowns [11][12]. In particular, one study shows that two-thirds of communication issues in a clinical setting are related to handoffs [13], indicating the importance of handoffs to not only the work continuity, but to the quality of patient care and patient safety. Because of that, how to ensure the continuity of care during handoffs is key to the quality of medical practices, issues such as communication patterns [6][10][14], information flow [5][15], artifacts usage, [5][6] as well as the sense-making process during handoffs [3] are explored in previous literature.

$\mathrm{HCI} / \mathrm{CSCW}$ field take handoffs, like shift changes, as a temporal rhythm that spins in the process of medical work and leads to information breakdowns in team collaboration [8]. Other HCI studies show that nurses spend almost an entire hour preparing for shift changes. They often deploy electronic-, verbal-, and paper-based artifacts to facilitate the information transition during shift changes [5] [6]. Randell et al. argues that in addition to handoffs, cognitive artifacts, e.g., the doctor's list and the white board, could provide continuous awareness among clinicians and consequently remove the tension away from handoffs transition moments [16]. Using cognitive artifacts to provide continues awareness suggest a new possibility of how handoffs could be handled beyond guiding the transition moments.

Beyond handoffs, other types of non-working moments may also happen when work efficiency is "impaired by over work, tiredness, even boredom derived from the work [17]." Understanding how other non-working moments are coordinated and covered is important for designing information systems to support continuous coverage in hospital work. This important aspect of work practice, however, has not yet been reported in the current HCI literature.

\section{Methodology}

This study took place at an Emergency Department (ED) in a large regional hospital. The ED service is generally non-stop, serving on average 200 patients every day. This high patient volume helps produce circumstances of continuous service. The ED is equipped with a centralized Electronic Medical Record (EMR) system for the purpose of keeping patient records as well as facilitating clinical work practices.

\subsection{Study Site: Main ED Unit}

There are 4 major units in the ED: a pediatric ED, an urgent care unit, a trauma center, and a main ED unit. Each unit has 8-16 patient rooms for the types of patients it serves. Beyond the four major patient treatment units, there is also a waiting room, triage rooms, an admitting office, a meeting room and an administrative office in the ED. Due to the size of the entire ED, this study took place primarily in the main ED Due to the size of the entire ED, this study took place primarily in the main ED area, where most of the attendings, nurses and ED technicians, and other ED staff, such as discharge manager, social worker, nursing manager and admin personnel are located. The main ED has 2 nursing stations and a MD station, surrounded by 16 patient rooms (see Figure 1). 


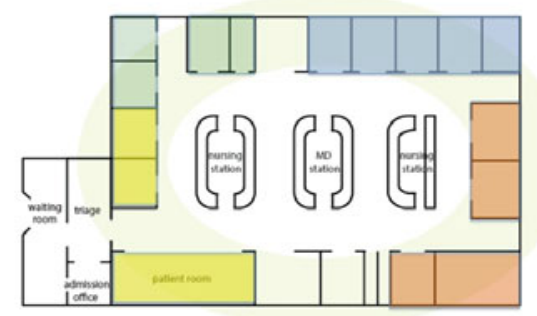

Fig. 1. The main ED map with the work locales of nurses. Four room nurses are each represented in one color and the float nurse is covering for the entire ED.

\subsection{Participants}

The ED has a total of 57 clinical staff members, with some non-clinical staff. Studies were conducted in two phases. Initially, we observed general ED work in public areas such as the meeting room, the MD station, waiting rooms, nursing stations and hallways. These observations covered most of the ED staff, and they helped us to gain an understanding of overall ED patient care. The second phase consisted of shadowing individual doctors and nurses working in the main ED area, triage room, and waiting rooms. For the research reported in this study, we shadowed 5 attending physicians, 5 room nurses, 4 triage nurses, and 2 float nurses. Other ED staff such as admission personnel, social workers, and the discharge manager were also observed, but are not reported due to the study's emphasis on the continuous coverage of patient care.

\subsection{Data and Data Collection}

A total of 120 hours of observations were conducted over a period of eight weeks, with 40 observation-hours dedicated to understanding how patient care is performed in the ED in general, and 80 hours of shadowing individual ED staff. Observations were divided into 4-5 hours long sessions where each session focused on one ED staff or location. During observations, we tracked down important incidents happening in the patient care process and asked ED staff to explain their work practices when patients were not around. We also attended daily nursing shift meetings. Observation notes were jotted down using paper and pen on site, and transcribed in detail later.

To ensure accurate representation, approximately half of the observations were carried out during ED peak times - weekends and Monday nights. In addition, we shadowed all roles involved in direct patient care, e.g. triage nurses, room nurses, ED doctors and waiting room nurses, as well as, all major locations on the ED floor, e.g. patients' waiting rooms, triages, nursing stations, MD stations and patient rooms. To protect patients' confidentiality, we stayed outside patient rooms when shadowing room nurses. However, since the EMR workstations were mounted at the corner of the patients' room facing outside, communication and documentation patterns could easily be observed without interfering with direct patient care. 


\subsection{Data Analysis}

After continuous coverage was identified as the main theme of this paper, instances that related to covering and handoffs activities were extracted from the observation notes. These activities were further categorized into three types according to their length and properties. It is notable that the findings in this study are not drawn from one individual ED staff; rather, they are based on a synthesis of the recurring behaviors demonstrated by multiple staff and repeated observations.

\section{Findings}

This section first introduces the key staff involved in the process of handoffs, and how their intertwined roles result in "continuous coverage;" then we describe shift handoffs as conducted through the EMR system and outline three types of inshift handoffs and articulate how they are coordinated on the ED floor.

\subsection{Patient Care in ED}

The continuous coverage of patient care starts as soon as patients arrive in the ED, where they are monitored in the waiting room and the triage rooms. Once patients are assigned a patient room, they are treated by a room nurse and an ED doctor. We use the term work locale to represent the physical coverage of ED staff's service area.

ED Doctors. The ED doctor's work largely consists of what Strauss calls “therapeutic work" [17]. ED doctors choose patients based on medical urgency, patient volume and their own expertise. Patients waiting to be seen by the doctor all reside in ED patient rooms. Each doctor, on average, handles 6-7 patients during the ED peak time and fewer patients during non-busy hours. After taking on a new patient, the ED doctor first travels to the patient room to do initial diagnosis work, e.g. interviews, physical exams, and then returns to the MD station to place orders in the EMR system, such as medications, procedures and labs. Orders will be sent to room nurses as pending tasks in the EMR. Doctors spend most of their time checking nursing notes, waiting for patient lab test results, discussing cases and writing progress notes at the MD station.

The ED doctors' work locale extends to the entire ED floor, since they do not pick up patients according to their locations. Doctors may travel to the furthest patient room or take care of a patient right across the MD station. Their movements in the ED are largely centered around the MD station, although they also work on all four ED units. In other words, a doctor's work does not anchor them at bedside at all times. When urgent situations occur, doctors can be paged through either a personal page or an overhead page announced to the entire building.

Room nurses. Each room nurse manages four patients' rooms in the ED. Nurses largely engage in the so-called "monitoring work" [17]. The reason we refer to room nurses" tasks as "monitoring work" is that they station themselves at patient bedside, continually monitoring patients' status, such as watching out for patient situations, 
checking vital signs every two hours, updating patient information on the nursing notes section of the EMR, and administrating orders received from ED doctors.

Room nurses, as observed in this study, are always checking the EMR system to see if they have any pending orders and to update nursing notes after every procedure they perform, whether at the bedside or at the nursing station. Each room nurse's service area is marked on Figure 1 using different colors. As indicated on the map, patient rooms assigned to each nurse are all located at the same corner of the main ED. Room nurses divide their entire working hours among patient rooms and the nursing station near their assignments. The typical routine of the room nurse is to check patients, update information at the bedside computer, follow administrative orders, and then write notes at the nursing stations.

Unlike the ED doctors who work on the entire ED floor, the work of the room nurses remain situated around the area of their assignment. This is primarily due to the nature of the monitoring work that must be done at the patients' bedside. For instance, the four room nurses in the main ED area are each in charge of four patients' rooms and there is no overlap among their room assignments (see Figure 1). In other words, at any given moment, there is only supposed to be one room nurse for each patient room. Thus, the working locale for a room nurse is the fan-shaped area from the nursing station to their patients' rooms. Nurses rarely step out of their designated working locales because they need to be readily available to monitor patient situations and to respond to patients' requests. This continuous coverage occurs even during the non-peak time, since nurses have to be prepared to help with any sudden emergencies, such as patient transported by ambulances.

Float nurse. The float nurse is an indispensible role in the process of maintaining continuous coverage. During every shift, one nurse is assigned the role of float nurse by the nursing manager. A float nurse receives no particular assignment, but covers for a room nurse whenever they leave their designated working locale during a shift, or when a room nurse needs help with a heavy workload.

A float nurse's working locale includes all the places that need covering, which in most cases, is the entire ED. Differing from doctors who mostly reside in their own station, a float nurse does not have a fixed working station, and is always moving around on the floor during a shift. Float nurses may actively approach room nurses to see if they want to be covered, or may wait to be called on for help.

\subsection{Continuous Coverage in the ED}

In the ED, a patient has to be continuously covered on both the therapeutic level and the monitoring level. Before the introduction of the EMR, doctors and nurses had to constantly communicate. Nowadays, doctors and nurses do not have to verbally update each other, since all work is communicated through the system. When an ED doctor signs an order for a patient in the EMR, the room nurse for this patient is automatically alerted. Similarly, when the room nurse of this patient updates notes in the system, the doctor can see them immediately at the MD station. Sometimes, doctors may not even notice who the room nurse of a particular patient is, since their working locale can be across the entire ED, and every patient of theirs may have a different room nurse. 
ED doctors are considered on duty as long as they stay in the hospital area, and have no need to be covered by others. In contrast, room nurses have to stay close to patient rooms for their entire service period in order to monitor patients (with the exception of the float nurse). Each work area only has one nurse, so whenever a nurse steps out to get water, take a brief break or have lunch, a disruption in the continuous coverage of patient care occurs; to avoid this, the room nurse needs to be covered before they can leave. In other words, an in-shift handoff is needed.

What follows are our findings regarding handoffs. First, we describe end-of-shift handoffs using the EMR system. Second, we detail three types of in-shift handoffs and their non-EMR-oriented coordination processes.

\subsection{End of Shift Handoffs through EMR}

Unlike nursing shift meetings in in-patient units that take more than 30 minutes [5] [6], the shift meetings in the ED usually last only 2-3 minutes and are held in the locker room next to the main ED area. Attendees of these meetings are all the incoming shift nurses and the nursing manager on duty. The locker room is where the incoming nurses first arrive when they start their workday. During the meeting, the nursing manager brings in a paper-schedule and announces each incoming nurse's assignment. A typical assignment looks like this ${ }^{1}$ : Mike - triage room 1, Melissa float nurse, Rowena - room 12-16, Lisa - room 16-20 and so forth. The nursing manager will then let each nurse know the time of his or her assigned lunchtime - the one-hour middle-of-shift meal. If there are no questions regarding the assignments, the nurses walk to their designated work locales to start their work. The earlier shift nurses in the ED never attend the shift meetings, but wait at their work locale until the incoming shift nurse arrives. Hence, nursing handoffs in the ED are all conducted at the local level where two shift nurses assigned to the same area hand over jobs at the working locale.

Since the deployment of the EMR system, the handoff between nurses now occurs in two stages: an informal verbal information handoff at patient bedside and a formal system handoff at either bedside or the nursing station. During the handoffs, the leaving nurse will walk through each patient room with the incoming nurse. For each room, the leaving nurse will first acknowledge the patient that the incoming nurse will take over, and then talk to the incoming nurse about this patient's situation outside the room. This step largely constitutes the act of passing patient care information to the next shift nurse as a means to maintain continuous care for the patient. After the verbal handoff, the leaving nurse signs off on their patients to the incoming nurse via the EMR system. After the formal handoff, the four patients automatically appear under the in-coming nurse's name when they first sign into the EMR system. The formal EMR signoff transfers the patient records and the responsibility of patient care officially to the in-coming nurse, as well as pending orders and other unfinished tasks.

\subsection{Three Types of In-shift Handoffs without EMR}

Handoffs not only occur between two shifts; they also frequently occur during the same shift, as a way of maintaining continuous coverage in ED care. In-shift handoffs

\footnotetext{
${ }^{1}$ All the names used in this paper are pseudonyms.
} 
are usually short-term, ranging from a few minutes to one hour. Unlike per-scheduled shift changes that occur in 12-hour intervals, in-shift handoffs are more spontaneous and rely on the interpersonal coordination among collocated nurses. This section describes how these in-shift handoffs occur and are coordinated on the ED floor.

Lunch breaks - Planned One-hour Coverage. Perhaps the most important task for a float nurse is to cover for lunch breaks, since nurses cannot work continuously for a 12 hours without taking a meal. "Lunch" is an hour-long time off in the middle of a shift, no matter if it is at midnight or in the early morning. Lunchtimes are prescheduled by the nursing manager and announced in the shift meetings. To maintain continuous coverage with only one float nurse, room nurses working the same shift are all assigned with different lunch times. As a result, lunch can happen anytime during a shift.

The float nurse moves to different working locales, covering lunch-breaks based on the nursing schedule. Although the lunch break is only an hour long, necessary patient information must still be handed over to a float nurse so that patient care tasks can be performed. During the handoff, room nurses go through their patients one by one, and remind the float nurse of tasks to be done in the following hour, e.g. the next two hour vital sign check up and monitoring for chest pains. This lunch hour handoff is similar to the "verbal handoff" that is performed at the end of shifts. Through this brief faceto-face conversation, the float nurse receives critical information about a patient and is given the key tasks that might be happening in the next hour.

Unlike end-of-shift handoffs, handoffs for lunch are transitioned only through verbal communication, but are not signed in to the EMR system. The consequence of this action is that while the room nurse is on break and is no longer the one caring for the patients, in the EMR system, the room nurse is still "in charge." Orders signed from ED doctors are still routed to the room nurse, but not the float nurse who is caring for the room nurse's patients. Since the EMR system is viewable to every ED staff, the float nurse could always check patient's information in a patient's records. Nevertheless, orders are not publicly accessible to everyone. However, what is justly notable in the lunch hour handoff is how the information and the responsibility of patient care are separated due to the lack of EMR-based signoffs. The lack of EMR based care transitions may result in serious consequences to medical work.

Ad Hoc Breaks - 10 Minutes Unplanned Coverage. The intensity of ED work may exhaust room nurses, potentially inducing errors and mistakes. Each room nurse is allowed to have approximately 3 ad hoc breaks per shift when and if they feel the need, each lasting for 10 minutes. While room nurses are not expected to take too many breaks, they are not expected to work during extreme tiredness either. The 10minute absence from their patients' bedside, though brief, still disrupts the continuous monitoring work that room nurses engage in, forcing them to find coverage for their ad hoc breaks via a float nurse. Similar to the lunch breaks described in the previous section, ad hoc breaks are negotiated verbally, and are not officially transferred in the EMR system.

The spontaneous nature of ad hoc breaks makes planning for them impossible, since the vagaries of tiredness are situated in the nature of the workday. This makes the coordination process of ad hoc breaks more challenging to perform in the ED. In an ideal situation, when a nurse wants to take a break, they should be able to contact 
the float nurse right away and immediately arrange for them to cover their patients. In reality, ad hoc breaks are usually initiated by the float nurse due to the lack of communication among nurses.

Ad hoc breaks are managed through a flow sheet placed on the left nursing station in the main ED area, where everyone can see it. The flow sheet is a table-lookschedule that is filled out during the shift. On the flow sheet, there is one line for each ED nurse, with the first column showing the shift time of the nurse, followed with their name, lunch break time, three ad hoc break times, and a note section.
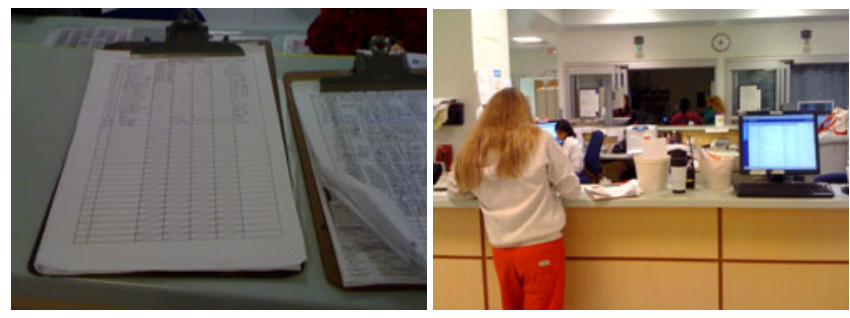

Fig. 2. The coordination of ad hoc breaks in the ED. Left, the flow sheet of nursing work; right, the float nurse is checking the flow sheet.

To coordinate ad hoc breaks, the float nurse checks the flow sheet constantly and speculates whether a room nurse may need an ad hoc break based on what has been documented on the flow sheet. For example, if nurse-A hasn't taken a break for the 4 hours since work began, and their lunchtime is schedule 3 hours later, the float nurse may consider giving nurse-A a break. Thus, the float nurse travels to nurse A's working locale and inquires whether a break is needed, and nurse-A will probably say yes given the circumstances. After nurse-A's coverage, the float nurse will sign the time of this ad hoc break on the flow sheet for nurse-A. In this way, the flow sheet plays an essential role as a central coordination artifact that allows the float nurse to manage continuous coverage. The ad hoc breaks are mainly initiated by the float nurse and the need for an ad hoc break is decided based on the flow sheet.

Use of the flow sheet to coordinate ad hoc breaks allows the float nurse to check, arrange and record for whom and when they have covered. The flow sheet greatly benefits the float nurse's work, but not those in need of breaks on the ED floor who are accidentally overlooked. On more than one occasion, we observed that nurses working in their locales were unable to locate the float nurse at the time they needed a break. Consider the following scenario ${ }^{2}$ :

Mike, a room nurse stationed at one left corner of the main ED wanted to take a break. He is not feeling well today and has been working for 3 hours. He looked around every 5 minutes but didn't see the float nurse in the main ED area. Mike asked Lisa - the other nurse working in the same nursing station - whether she knows where the float nurse is. Lisa said, "Why don't you check the flow sheet and see where she is?" Mike ran to check the flow sheet on the other side of the main ED and was told by the nursing manager that the float nurse was covering a lunch break in

\footnotetext{
${ }^{2}$ Scenarios are all drawn from observation notes.
} 
the urgent care unit. Mike came back and asked Lisa if she could cover him for a few minutes so he could get a snack at the fitting room. Lisa answered, "let me finish this vital first and I can keep an eye on [your patients] for you."

This scenario indicates the inefficiency afforded by coordination of ad hoc breaks using only the centralized flow sheet. Many times during observations, we saw the float nurse standing at the nursing station examining the flow sheet and considering who should be given a short break. The need for ad hoc breaks is not merely based on the dimension of time. Issues such as an individual's physical condition, the busyness of ED work, the unit a nurse is in and the demand of the nurse's patients also affects ad hoc breaks. Certainly, working at a trauma unit would be more exhausting than working at an urgent care unit, and night shifts produces more tiredness than day shifts. Unfortunately, time is generally the sole criterion for deciding whom to give ad hoc breaks in the ED.

When nurses can't find coverage from the float nurse, they have to ask for help locally due to their inability to travel far from their work locale. From our observations, nurses were able to ask for help from other nurses they could physically see, but were less likely to seek help from the other ED units, or even the other side of the main ED unit. Every nurse could use the phone at their ED station to call others stations, and even page the entire ED. The triage nurse has a walkie-talkie to communicate with the nursing manager. Yet, the float nurse has no communication devices on-hand and consensus seems to be that the overhead pages, which are intended for services such as paging doctors, should not be used for solving this seemingly trivial issue, such as breaks due to the tiredness.

Team Nursing - Shared High Workload Coverage. Though continuous coverage is viewed as linear temporal coordination in medical work [1], during this current study, we witnessed that continuous patient care are shared among multiple room nurses simultaneously, as developed by the nurse on the ED floor in the so-called, "Team Nursing" practice.

Traditionally, one ED nurse takes care of four patients. It is assumed that a nurse can manage the orders; check-up and monitoring work for four patients simultaneously. Sometimes though, this assumption becomes problematic, as there is no coverage for emergency situations. As one nurse told us, "all of them [patients] are stable right now. No one knows what might happen [the] next minute; if two breakout at the same time, I am not gonna cover both of them at the same time." Here, even the room nurse is physically at the working locale, yet, attention can only be given to one patient at a time, leaving the other three patients momentarily uncovered. The general assignment of the ED would allow a nurse to cover four patients on a regular basis, but not when two or even more patients need to be attended to at the same time.

Helping with the room nurses' temporarily heavy workload is theoretically one of the daily duties of the float nurse. However, situations wherein the room nurse is physically present but is unable to give the needed attention to a patient tend to be urgent, and though the float nurse could be called, there probably would not be enough time for the necessary patient information to pass from the room nurse to the float nurse. In a new strategy developed by nurses at our field site called "team nursing," nurses cover locally for each other in cases such as these. 


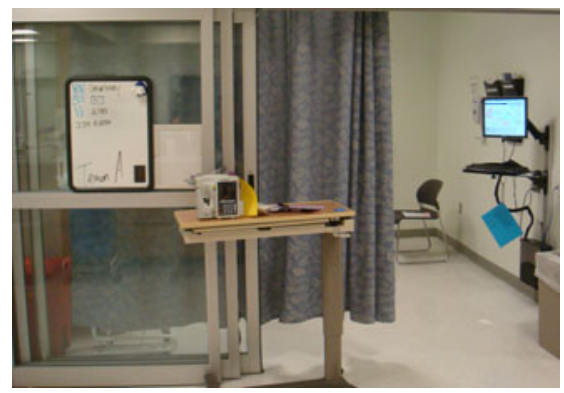

Fig. 3. The whiteboard hung outside each patient room. It shows the room nurse belongs to "Team A".

"Team Nursing" pairs nurses into teams based on their physical proximity. This physical proximity allows nurses to cover each other when needed. In "Team Nursing," however, nurses do not only provide coverage when urgent situations break out; instead, nurses are asked to stay aware of the situation of all the patients of all the nurses on their team. For example, the two nurses in Team A work in the left nurses' station, and have eight patient rooms collaboratively managed between them. 'Team Nursing' is useful because, as one room nurse explained, "if anything happens, we know why this patient is here and what happened to him, sometimes a few seconds can save a life." When all team members are constantly check of each other's patients, the verbal handoff can be avoided when an urgent situation arises. In addition, team members are able to gain an understanding of all the patients in their team gradually and thoroughly over time - an understanding that is not possible for a float nurse to obtain even through a verbal handoff.

"Team Nursing" provides room nurses with a way to avoid possible information breakdowns during emergency situations. Nurses give and receive constant patient updates, and read the charts of each other's patients. Through team nursing, nurses can avoid the common practice of verbally reporting the patient situation to the covering nurse during a time of urgency. As one of the room nurses we spoke with commented in the previous paragraph, a matter of one second can save, or lose, a life, and by shaving away the time a handoff takes, precious time is added to direct patient care. With more than one nurse paying attention to a patient, it is also likely that potential medical errors could be avoided through the overlapped attentions.

Nevertheless, in 'Team Nursing,' each nurse still receives their own assignments and there is no cross-coverage among these assignments. The 'teams' of nurses only exist on the whiteboard; nurses still act solely in the EMR system. What is shared in "team-nursing" is the responsibility and information of a pool of patients. Each nurse gains not only a sense of partial responsibility for all the patients of their team, but also for the nurses who are their team members. It is also notable that the creation of nursing teams is coordinated mainly through spatial proximity, and not a linear temporal arrangement. 


\section{Discussions}

This section takes a synthesized view towards in-shift handoffs. First, we discuss how in-shift handoffs are managed through the interplay of temporal and spatial coordination on the ED floor. Second, we highlight the issues resulted from the lack of in-shift handoffs in system design.

\subsection{The Coordination of In-shift Handoffs}

Though the continuous coverage concept applies to nurses and doctors, in-shift handoffs only occur among nurses, and not ED doctors. In this section, we discuss how in-shift handoffs are coordinated by nurses collocated on the ED floor.

Working Locale Attachment. As evident in our study, in-shift handoffs apply solely to nurses, but not ED doctors. Doctors rarely seek coverage if they need to get lunch outside the ED or leave for 5 minutes. This is largely due to the nurses' attachment to a specific working area. The doctors' work locale, as described in the ED work section, is the entire ED. Doctors accept patients from any place in the ED and remain in the MD station for most of their shift. Because the doctor's work is not attached to a specific patient room, there is no such notion of leaving patients for a short break, and consequently, no need for in-shift coverage.

The strong attachment nurses exhibit to their working locales can even be seen in the way that their daily assignments are given; nurse are never assigned to patients, but room numbers. Spatially, the room nurses manage neighbor each other and the nursing station they use is right outside their patient rooms. This setup illustrates how nurses need to be physically present to maintain the continuous coverage of their patients - in other words, nurses have to be covered as soon as they step out of their designated working locales. In this sense, taking lunches downstairs at the cafeteria is an absence from work, as is drinking water at the locker room next door, or even going to restroom. All of these require nurses to find short-term replacements.

Only one of the three types of in-shift handoffs noted in this paper can be anticipated: lunch break handoffs. The other two forms of coverage, related to ad hoc breaks and high workloads, are more spontaneous in nature and are usually coordinated at the local level. Interestingly, no matter how long the coverage is, it inevitably involves handing patients from one room nurse to another, and the consequences for one 5-minute break is similar to a full shift handoffs. The current system design lacks sufficient acknowledgment of these short-term handoffs, potentially leading to other types of handoff breakdowns.

From Temporal Transition to Local Awareness. More importantly, the in-shift handoffs identified in this study are often coordinated locally by nurses working at nearby locales. This coordination of in-shift handoffs shows that the scheduling process of handoffs is not merely a temporally oriented task, but also one that takes place at the locations where nurses engage in work.

Intershift level coverage is coordinated through rotations of temporal rhythms in medical work [8]. Rhythms such as shifts follow a strict 12-hour timeline and are a linear process of one after another. There is generally no overlap between two shifts other than the brief transition period. In contrast, among the three identified in-shift 
handoffs, only the lunch break can be scheduled since it occurs only once during the shift, and has to be spread out to different times (once for each nurse) for the sake of scheduling. The other in-shift coverage are more spontaneous in nature since it is not easy to predict when a nurse may need an ad hoc break due to tiredness or when an extra hand will be needed due to medical urgency. The more unpredictable a break is, the more unlikely it is to schedule the coverage in advance via temporal coordination.

As described in the findings section, ad hoc breaks are covered mostly through spatial arrangements, as opposed to temporal coordination. For ad hoc breaks, the nursing manager allows each nurse to have approximately 3 short breaks during their shift. Yet, the specific times that a nurse requires a break cannot not be scheduled, and the float nurse may not be available or be able to notified when a room nurse need it. As a result, nurses working close by may be asked to cover for a break when the float nurse cannot. This is an example of a local coordination mechanism between nearby working locales that enables continuous coverage in the ED.

In addition, coverage of urgent situations by "Team Nursing," designed at the field site we worked at, also reaches beyond the linear temporal coordination that most studies previously reported. Nurses in a team cover patients not in the "one-leave-onein" mode. By overlapping their coverage for a single patient, each nurse still has their own assignments, but stays aware of all the team's patients so that in times of urgency, there is no need to go outside the team for coverage. This pattern switches the linear coverage into the paralleled overlap coverage. It is important to note that this parallel coverage is also enabled through the spatial coordination within the collocated nursing teams.

Just as Randell argues for the use of cognitive artifacts to help physicians stay aware of patients at all times, as opposed to handing jobs over just during the short handoffs periods, local awareness also facilitates the continuity of patient coverage and can alleviate the sudden "drop the baton" situations that can happen during transition moments [16]. In 'Team Nursing," nurses in the same team know each other's patients naturally through their physical proximity. Many times in the study, we saw the nurses asking about the status of another team member's patients, or even sharing their patient care information through casual chats at nursing stations. When this does not happen naturally, "Team Nursing" practice requires nurses in the same team to update each other on patient cases throughout the entire shift, and to constantly check up on each other's patient charts in the EMR. So when it comes to managing in-shift handoffs through local coordination, it is likely that the nurses working nearby would already have certain prior knowledge about each other's patients. Thus, the burden of in-shift handoffs is no longer in the short transition period, but accumulated over time through constantly updating and acknowledging patients information. From this sense, the local awareness is the knowledge of things going on in their local area of neighboring patients. Indeed, this local awareness is a continuous endeavor rather than a sudden transition. Even though the actual handoff may still happen in a short period of time, the information needed for patient care is updated gradually as a shift progresses. 


\subsection{The Impact of In-shift Handoffs}

Much of medical work is now mediated through the use of the EMR systems. Consequently, the system also impacts how handoffs are conducted on the nursing floor. In this section, we discuss the potential impact caused by the lack of the proper EMR-based in-shift handoffs on the ED floor.

The Lack of System Supported In-shift Handoffs. Previously we used the definition of "transition of information, responsibility and authority" between two people to refer to handoffs in the medical field. Zerubavel [1] also emphasized the importance of responsibility in the handoff process. In previous studies, the layers of handoff, such as responsibility, information and artifacts, are transitioned simultaneously as nurses pass over to the next shift [5][6]. However, handoffs in the ED take place in twophases: first, passing over authority and information at the patient bedside, and second, officially signing-off responsibility in the EMR at the nursing station. Both phases are essential for the quality of patient care and are necessary steps to go through, since the EMR is not only a patient record system, but also a document of work activities that could be for liability purposes e.g. [18][19].

Nevertheless, most short-term handoffs only go through the initial stage of verbal transitions, but not in the information system. This is partially due to a lack of design to support short-term coverage in the information system. As is, the handoff process now is so complex, nurses would assume it acceptable not to signoff, and continue to find a nurse to cover for a few minutes. Similarly, "Team Nursing" only exists on the whiteboard hanging outside the patient rooms, and not in the EMR system. In this case, responsibility of a patient still falls completely on the room nurse, despite the rest of "Team A" checking the patient's chart in the EMR and caring for the patient when emergencies happen.

The lack of formal records for short-term handoffs bears various consequences for medical work. Nowadays, the EMR is not only a database of patient records, but serves as a central system of supporting all types of clinical work [20]. Evidence shows that the EMR is increasingly used to trace clinical activities for work efficiency, medical errors and responsibilities [18][9]. The lack of EMR-based transitions during temporary handoffs may lead to mismatch between the actual work practice and the records of work practices stored in the system. For instance, the covering nurse's tasks will not be documented in the formal medical records. When these tasks are checked afterwards, the actual nurse who performed the tasks and the nurses who is indicated in the system as the task handler will not be the same person. Also, it is arguable that many breaks are not documented properly in the flow sheet since they are handled locally without leaving any records with the nursing manager. This is not only legally conflicting, but also challenging to the ED management since the manager is now oblivious to how many breaks a nurse takes in a shift - key information that needs to be kept track of at management level.

Illusion of Communication. Since the deployment of the EMR system, most patient care information is mediated through the information system. Orders from doctors are routed 
to the "charge" nurse automatically. ${ }^{3}$ The charge nurse receives these orders as pending tasks whenever they sign in to the system. Due to the busyness and multi-patient care of ED practice, doctors may not be able to follow up nurses in person with each order they prescribed, but just assume that these orders will be checked and performed as the earliest convenience on the nurse's end. Nevertheless, the lack of design for in-shift handoffs could potentially lead to delaying in receiving information in the ED.

Since orders are no longer passed along on paper or through phone calls, room nurses who are monitoring patients information have to check the EMR system frequently to see if there are any pending orders. As what we observed in the study, nurses always log into system every 2-3 minute to see if they have any new pending orders. This frequent checking ensures timely delivery of medications to avoid patient suffering and delay in care.

However, the lack of concern for in-shift handoffs may lead to delays in receiving patients' orders. Doctors who order a test or new medication may not know that a nurse is on a designated lunch break at the time they put in an urgent order. Similarly, the float nurse may not be aware of a pending order since the order is only routed to the room nurse who is on break. This delay in receiving information has been referred to as illusion of communication in previous literature [21]. The illusion happens when one assumes communication is happening after orders are put into the system, but the orders may not be checked in the system by the other. This generally is not an issue for ED nurses, as they frequently log in and check the pending orders every a few minutes. But since the orders are not marked as pending orders in the float nurse' to do list, it may be overlooked during the in-shift handoffs. From this sense, the illusion of communication is partially due to the lack of support for in-shift handoffs in the information systems.

\section{Implications for System Design}

The findings of this study suggest that continuous coverage should be considered a primary design principle for clinical systems. Drawn from our qualitative field observations in the ED, we found that continuous coverage is maintained not only through the handoffs between two shifts, but also through various non-working moments that occur during the same shift of work. The lack of a proper design for these in-shift handoffs may impact both nursing work and the nursing/doctor collaborations that are mediated through the information systems. In this section, we outline possible design opportunities based on the findings of the study. Noteworthy is that what we suggest here is by no means the only design solution. Rather, it is an implication leading to the designs of clinical systems that take in-shift handoffs into considerations.

\subsection{Easy Role Switch During In-Shift Handoffs}

As described earlier, only at the end of shift do nurses sign-off patients officially in the EMR system; other in-shift handoffs are conducted verbally. Indeed, if the

\footnotetext{
3 This implication is based on the observations from the current study. This may not be applied to other EMR systems or other emergency departments.
} 
in-shifts handoffs were handled in the system, nurses would have to sign patient to the covering nurse first, then sign it back a few minutes later. The complexity and extra procedures involved in the EMR-based signoff keeps nurses from doing it during temporary breaks. This suggests that end-of-shift handoffs and in-shifts handoffs should be handled differently in the EMR system. System design should allow for easier role transition during temporary coverage periods, such as providing a covering feature to allow a one-click temporary signoff during in-shift handoffs, or add float nurses as a covering person in the system and allow role transitions for the one being covered directly. Simplifying the system signoff procedures would encourage clinicians to use it for the brief in-shift handoffs process and to avoid the potential communication illusions and delays.

\subsection{Social Display of In-shifts Handoffs}

Clearly indicated in our study, ED clinicians work in different working locales without much overlap. Room nurses stay nearby the patient rooms they manage, and ED doctors work primarily at the MD station. Nevertheless, the status of nurses and the orders doctors prescribed have to be coordinated during the in-shift handoffs. We suggest the EMR system to incorporate a social display system in order to provide awareness information for clinicians working on the ED floor. The display system can mark the status of each nurse and new orders for each room using various color schemes on a shared ED floor map. This map is then shown on the EMR front page or even projected onto a larger screen that can be publicly seen on the ED floor. In doing this, everyone in the ED can be aware of each other's working status, like who is on break (e.g. in yellow color) and which patient has new orders (e.g. in red color). In this case, the illusions of communication can be eliminated since new orders will be visible without logging onto the EMR system. Doctors could also be cautious when they notice the room nurse is on break. Nurses can also remind each other as the colors associated with patient room is publicly displayed. Using the display system can benefit the communication between doctors and nurses in general, since by flagging new orders in the social display system, room nurses no longer need to log in to the EMR every 2-3 minutes to see if they have pending orders.

\subsection{Shared Responsibility}

Different from the temporal work coordination that occurs at the end-of-shift handoffs, in-shift handoffs are often managed through spatial coordination among nearby nurses. That is, nurses working nearby act as a team to help each other when necessary. The local coordination turns the solo practice of "one nurse cares for four patients" into a collaborative practice of "three nurses in team A manage twelve patients together." To do so, nurses in a team maintain a local awareness by constantly checking on and updating patients' situations with each other. We suggest the design of patient care systems take advantage of local awareness and provide shared responsibilities for nurses working nearby. This way allows team nursing to be formalized in the medical records. Team members could not only have access to patient information, but are also able to document and order in the records. In this case, the medical record become a cognitive artifact [16] that provides constant 
information sharing for in-shift handoffs and relieves the possible "baton" drop transition moments.

\section{Conclusion}

In this study, we explore how the concept of continuous coverage is practiced in an emergency department. Our observations reveal patterns of unreported short-term handoffs in the same shift of nursing care. These in-shift handoffs result from the strong attachment of nursing work to the working locale they are assigned. Differing from previous studies on handoffs, this current study shows that when handoffs occur within the same shift, they are more likely to be coordinated through local arrangement among collocated nurses. The handoffs that are arranged through the closeness of working locales enable constant updates among nurses and provide them a sense of local awareness - an awareness of knowing patients situations in the nearby rooms. This mechanism could avoid the sudden breakdown in the normal handoff processes. However, since these short term handoffs are hidden in the same shift work and are not recognized by system designers, they may lead to issues such as the separation of information and responsibility in patient care, and the illusion of communication, if they are not properly handled. The findings of this study call attention to the design of systems that account for in-shift handoffs that can improve handoff processes via the coordination of local awareness during ED work. In-shift handoffs may also exist in areas that require strong attachment to an employee's working locale, such as space shuttle, software design, and 24/7 services areas.

Acknowledgements. We would like to thank Drs. Harris R. Stutman, Giancarlo P. DiMassa and ED staff at Long Beach Memorial Medical Center. Thanks to Victor Ngo for helping proofread the earlier draft of this paper.

\section{References}

1. Zerubavel, E.: Patterns of time in hospital life: a sociological perspective. University of Chicago Press, Chicago (1979)

2. TeamSTEPPS.: Team Strategies and Tools to Enhance Performance and Patient Safety: Pocket guide (AHRQ Pub No 06-0020-2). Agency for Healthcare Research and Quality, Rockville, MD (2006)

3. Sharma, N., Cohen, M., Hilligoss, B., Patterson, E.: Handoffs \& Handovers: Collaborating in Turns. In: CSCW 2010 Workshop, Savannah, Atlanta (2010)

4. Wilson, S., Galliers, J., Alem, L.: Handover: Collaboration for Continuity of Work. In: ECSCW 2007 Workshop, Limerick, Ireland (2007)

5. Tang, C., Carpendale, S.: An Observational Study on Information Flow during Nurses' Shift Work. In: Proceedings of CHI 2007, pp. 219-228 (2007)

6. Zhou, X. Ackerman, M.S., Zheng, K.: I just don't know why it's gone: maintaining informal information use inpatient care. In: Proc. CHI 2009, pp. 2061-2067 (2009)

7. Berg, M.: Accumulating and Coordinating: Occasions for Information Technologies in Medical Work. Computer Supported Cooperative Work 8(4), 373-401 (1999) 
8. Reddy, M., Dourish, P.: A finger on the pulse: Temporal rhythms and information seeking in medical care. In: Proceeding of CSCW 2002, pp. 344-353 (2002)

9. Bardram, J.E., Bossen, C.: Mobility Work: The Spatial Dimension of Collaboration at a Hospital. In: Proceedings of the ACM CSCW 2005, pp. 131-160 (2005)

10. Patterson, E.S., Roth, E.M., Woods, D.D., Chow, R., Gomes, J.O.: Handoff strategies in settings with high consequences for failure: lessons for health care operations. Int. J. Qual. Health Care 16, 125-132 (2004)

11. Friesen, A.M., White, V.S., Byers, F.J.: Handoffs: Implications for Nurses, in Patient Safety and Quality: An Evidence-Based Handbook for nurses. AHRQ (2008)

12. Gandhi, T.K.: Fumbled handoffs: Oned ropped ball after another. Ann. Intern. Med. 142, 352-358 (2005)

13. Ebright, P.R., Urden, L., Patterson, E.: Themes surrounding novice nurse near-miss and adverse-event situations. J. Nurs. Adm. 34, 531-538 (2004)

14. Haig, K.M., Sutton, S., Whittington, J.: SBAR: a shared mental model for improving communication between clinicians. Jt Comm J. Qual. Patient. Saf. 32, 167-175 (2006)

15. Sarcevic, A., Burd, R.S.: Information handover in time-critical work. In: Proceedings of the GROUP 2009, pp. 301-310 (2009)

16. Randell, R., Wilson, S., Woodward, P., Galliers, J.: Beyond handover: supporting awareness for continuous coverage. In: Cognition, Technology \& Work (2010)

17. Strauss, A., Fagerhaugh, S., Suczek, B., Wiener, C.: Social Organization of Medical Work. University of Chicago, Chicago (1985)

18. Ash, J.S., Berg, M., Coiera, E.: Some unintended consequences of information technology in health care: the nature of patient care information system-related errors. J. Am. Med. Inform. Assoc. 11, 104-112 (2004)

19. Koppel, R., et al.: Role of Computerized Physician Order Entry Systems in Facilitating Medication Errors. JAMA 293, 1197-1203 (2005)

20. Heath, C., Luff, P.: Documents and Professional Practice: 'bad' organizational reasons for 'good' clinical records. In: Proc. CSCW 1996, pp. 354-363 (1996)

21. Dykstra, R.: Computerized physician order entry and communication: reciprocal impacts. In: Proc. AMIA Symp., pp. 230-234 (2002) 